\title{
Expression of heparanase regulates cell proliferation, apoptosis, migration and invasion in vitro in papillary thyroid carcinoma cells with multiple signaling pathways involved
}

\section{Chuanjia Yang}

the Affiliated Shengjing Hospital of China Medical University

Siyang Zhang

China Medical University https://orcid.org/0000-0003-2757-8809

\section{Xiaoying Chang}

the Affiliated Shengjing Hospital of China Medical University

\section{Yonglian Huang}

the Affiliated Shengjing Hospital of China Medical University

Dongxu Cui

the Affiliated Shengjing Hospital of China Medical University

Zhen Liu ( $\square$ liuzhen1973@aliyun.com )

https://orcid.org/0000-0001-5338-7219

\section{Research article}

Keywords: heparanase, papillary thyroid carcinoma, proliferation, apoptosis, migration, invasion, signaling pathways

Posted Date: August 20th, 2019

DOI: https://doi.org/10.21203/rs.2.13185/v1

License: (c) (i) This work is licensed under a Creative Commons Attribution 4.0 International License. Read Full License 


\section{Abstract}

Background Heparanase (HPSE) is an endo- $\beta$-D-glucuronidase, which is found overexpressed in various human cancers. The purpose of our work was to investigate the possible role of HPSE and the involved signaling molecules in the development of papillary thyroid carcinoma. Methods In this study, the expression plasmid of HPSE and RNA interference with shRNA specific for HPSE were used to elucidate the effects of HPSE on proliferation, apoptosis, migration and invasion in papillary thyroid carcinoma cells of B-CPAP. The probable downstream signaling molecules of HPSE were also explored. Results The results showed that upregulation of HPSE significantly promoted cell proliferation, migration and invasion of B-CPAP cells, and interfered with cell apoptosis. On the contrary, knockdown of HPSE exhibited the opposite effects. Compared with the parental cells, HPSE silencing cells showed attenuated capabilities of proliferation, migration and invasion, yet the apoptotic rate of transfected cells was increased. The activations of various signaling molecules correlated with cell biological behavior were found to be regulated by HPSE upregulation or knockdown. Conclusions Our results suggested that HPSE probably contributed to the progression and metastasis of papillary thyroid carcinoma, which were associated with multiple signaling pathways. HPSE could be a potent molecular target for the therapeutic strategy of papillary thyroid carcinoma.

\section{Background}

Heparanase (HPSE) is an endo-beta-glucuronidase and regarded to promote the invasion and metastasis of tumor cells. It has been confirmed that HPSE facilitates the proliferation and metastasis of ovarian cancer [1], and downregulation of HPSE resulted in the inhibition of adhesion and aggressiveness of hepatocellular carcinoma cells [2]. HPSE was also an important regulator in tumor microenvironment, such as tumor angiogenesis. The mRNA levels of HPSE were negatively associated with the prognosis factors of PDAC patients. HPSE influenced the expression of VEGF-C and promoted invasion in BxPC-3 cells [3]. It was found that HPSE are key proteins influencing tumor angiogenesis and cell proliferation and invasion in cervical cancer, possibly via the NF-KB signaling pathway [4].

Mechanism(s) underlying the progression of different tumors by HPSE regulation could be multifaceted. It was found that HCCLM3 cells with high HPSE expression showed higher trans-endothelial migration (TEM) rate. Downregulation of HPSE or inhibition its activity resulted in suppression of TEM in HCC cells [5]. A role for HPSE in modulating autophagy was established in normal and malignant cells, thereby conferring growth advantages as well as resistance to chemotherapy [6]. MiR-558 upregulated the expression of HPSE in gastric cancer cell lines. MiR-558 facilitates the progression of gastric cancer through directly targeting the HPSE promoter to attenuate Smad4-mediated repression of HPSE expression [7].

A study of thyroid carcinoma found that the expression of HPSE2 was increased and tumors showed a typical combination of positive labeling for neoplastic cells and negative immunostaining in colloid [8]. However, the function of HPSE and the underlying mechanisms in human papillary thyroid carcinoma 
have not been sufficiently disclosed and resolved. In this study, HPSE was upregulated or knocked down by HPSE plasmid or shRNA transfection to investigate the roles of HPSE in proliferation, apoptosis, migration and invasion of papillary thyroid carcinoma cells. Furthermore, the activity of downstream signaling(s) along with HPSE upregulation or knockdown was also explored.

\section{Methods}

\section{HPSE overexpression and RNA interference}

Human papillary thyroid carcinoma of B-CPAP cells were purchased from Chinese Academy of Sciences and cultured in RPMI 1640 medium (Gibco, USA), supplemented with $10 \%$ FBS, under the condition of $37^{\circ} \mathrm{C}$ and $5 \% \mathrm{CO} 2$. The overexpression plasmid for HPSE and the empty vector were purchased from GeneChem (China). The shRNAs specific for HPSE and the scrambled non-targeted shRNA were also purchased from GeneChem (China). All the plasmid were transfected into B-CPAP cells using Lipofectamine 3000 (Invitrogen, USA). The experiments for cells were repeated at least three times.

\section{Quantitative real-time polymerase chain reaction (qPCR)}

Total RNA was extracted using RNApure kit (Aidlab, China) and reverse transcribed into cDNA using GoScript Reverse Transcription System (Promega, USA). qPCR was performed using the GoTaq qPCR Master Mix (Promega, USA) in the Roche LightCycler 480 Real Time PCR instrument. Primers were used as follows: HPSE forward: 5'-AGT GGG TGT GGG TGA TTT CC-3'; reverse: 5'-GGC TCC TGG GTG AAG AAG TC-3'. GAPDH forward: 5'-CAG GAG GCA TTG CTG ATG AT-3; reverse: 5'-GAA GGC TGG GGC TCA TTT-3'. The length of PCR products were 193bp and 138bp, respectively. The qPCR was performed for 40 cycles with an initial denaturation at $95^{\circ} \mathrm{C}$ for $2 \mathrm{~min}$, amplification at $95^{\circ} \mathrm{C}$ for $15 \mathrm{~s}$, and annealing at $60^{\circ} \mathrm{C}$ for $1 \mathrm{~min}$. GAPDH was used as reference gene, and the results were analyzed using $2^{-\Delta \Delta C t}$ method.

\section{Western blot}

Cells were lysed in RIPA containing protease inhibitor (Promega, USA) and phosphatase inhibitor (APPLYGEN, China) cocktail, and centrifuged for the supernatant extraction. 50 $\mathrm{\mu g}$ total protein was used for electrophoresis and transmembrane routinely. Rabbit anti-HPSE (1:1000, PeproTech, USA), p-FAK (1:500, Sangon Biotech, China), p-PDK1, p-PKCa/ $\beta$ II, p-MARCKS (1:1000, Cell Signaling Technology, USA), p-Akt, cleaved caspase-3, cleaved PARP (1:1000, Beyotime, China) or mouse anti-p-ERK (1:200, Santa Cruz, USA), $\beta$-actin (1:500, ZSGB-BIO, China) antibodies were used as primary antibodies and incubated with membranes at $4^{\circ} \mathrm{C}$ overnight. Then the goat anti-rabbit (1:4000) or anti-mouse $\lg$ (1:2000, both from ZSGB-BIO, China) was used, and membranes were developed using ECL (Millipore, USA). The software of Image $J$ was used to evaluate the integrated optical density (IOD) of protein bands. The ratio 
of IOD (HPSE) and IOD ( $\beta$-actin) of the same specimen was calculated as the relative expression level of HPSE protein.

\section{EdU incorporation assay}

The kFlour555 Click-iT EdU imaging kit (KeyGEN BioTECH, China) was applied to explore cell proliferation. The staining was performed briefly as follows: cells were cultured with diluted EdU of $20 \mu \mathrm{mol} / \mathrm{L}$ for $2 \mathrm{~h}$. Cells were fixed with $4 \%$ paraformaldehyde, and neutralized by glycine solution of $2 \mathrm{mg} / \mathrm{mL}$. After washed with PBS, cells were permeated with $0.5 \%$ Triton X-100, and then incubated with prepared Click-iT reaction cocktail in the dark. Hoechst33342 was used to counterstain nucleus. Under an inverted fluorescent microscopy, 5 random fields of 200x were focused and cells with red or blue fluorescence were counted in the same fields. Cell proliferative rates were represented as red/blue.

\section{Cell apoptosis assay.}

The cell apoptosis was examined by flow cytometry using an Annexin V-PE/7-AAD apoptosis detection kit (KeyGEN BioTECH, China). Cells were digested and collected using trypsin without EDTA. Cells $\left(1 \times 10^{5}\right)$ were washed twice in PBS and incubated in mixture of $5 \mu \mathrm{L} 7-\mathrm{AAD}$ in $50 \mu \mathrm{L}$ binding buffer for $10 \mathrm{~min}$ in the dark. After gently mixed with $450 \mu$ l binding buffer, cells were added with $1 \mu$ I Annexin V-PE and incubated for $10 \mathrm{~min}$ away from light. Results are representative of three individual experiments.

\section{Wound healing assay}

Cell were passaged $24 \mathrm{~h}$ before scarification and seeded in 6 well plate $\left(1 \times 10^{6}\right) .200 \mu \mathrm{L}$ pipettes were used to draw a straight line, which was vertical to the bottom of plate, using ruler as a guide. The detached cells were removed by PBS. Cells in plate were kept on being cultured in medium without FBS and cell migration was monitored at $12 \mathrm{~h}$ and $24 \mathrm{~h} .5$ random fields of $100 \times$ were selected at $24 \mathrm{~h}$, and images were captured. The blank area $(A)$ in the scratch was measured using Image $\mathrm{J}$ soft, and the wound healing rate was calculated as (AOh-A24h)/A0h×100\%.

\section{Cell invasion assay.}

The cell invasion was performed in a transwell chamber (Costar, USA) of 24-well with an $8 \mu \mathrm{m}$ pore size insert, which was precoated with Matrigel (BD, USA). Cells $\left(1 \times 10^{4}\right)$ were seeded in the upper chamber, and cultured in medium with $1 \%$ FBS for $24 \mathrm{~h}$. Cells were allowed to migrate towards medium containing $10 \%$ FBS in the bottom chamber. Cells on the upper membrane surface were erased with a cotton swab, and 
the migratory cells attached to the lower membrane surface were fixed with $4 \%$ paraformaldehyde and stained with crystal violet. The number of invasive cells was counted in 5 random fields of 200x under a microscope. Data shown are representative of three individual wells.

\section{Statistical analysis}

The statistical software of SPSS 13.0 was applied to perform data analysis. One-way ANOVA was used to evaluate the differences between cells with various plasmid transfection. Multiple comparisons were made using LSD-t test within groups. All data were shown as mean $\pm S D$ and data were regarded statistically significant when the $p$

\section{Results}

The overexpression or knockdown of HPSE in MRNA and protein level were demonstrated by qPCR and western blot

Cells were transiently transfected with HPSE plasmid or shRNA, and the mRNA and protein level of HPSE were evaluated by qPCR and western blot. Our results showed that the expression of mRNA (Fig.1A) and protein (Fig.1B) in HPSE transfected cells were significantly upregulated, compared with contrl and empty vector groups. Moreover, the relative mRNA (Fig.1A) and protein (Fig.1B) level were apparently knocked down in HPSE shRNA transfected cells, in comparison with other groups.

\section{The effects of HPSE plasmid or shRNA on cell proliferation}

Cells were transiently transfected with HPSE plasmid or shRNA, and cell proliferation was evaluated by EdU incorporation assay. Our results showed that the percentages of EdU positive cells were $30.0 \pm 2.9 \%$, $34.0 \pm 5.2 \%, 44.2 \pm 5.5 \%$ and $27.6 \pm 4.1 \%$ in control, empty vector, HPSE plasmid and shRNA transfection groups, respectively $(p=0.00014)$. It was shown that cell proliferation was facilitated by HPSE transfection, compared with control or empty vector group. It was implied that cell proliferation was inhibited when HPSE was downregulated by shRNA transfection (Fig.2).

\section{The effects of HPSE plasmid or shRNA on cell apoptosis}

Cells were transiently transfected with HPSE plasmid or shRNA, and cell apoptosis was examined by Annexin V-PE/7-AAD double staining using flow cytometer. The apoptotic rate of cells with HPSE upregulation was $5.72 \pm 1.22 \%$, and the control and empty vector transfected cells were found a higher 
apoptotic rates of $9.28 \pm 1.04 \%$ and $8.40 \pm 1.27 \%(\mathrm{p}=0.024)$. In cells with HPSE knockdown, we observed the apoptotic rate of $17.01 \pm 1.43 \%$, which was higher that other groups $(p=0.0002)$. These results shown in Fig. 3 indicated that HPSE protected cells survival avoid of apoptosis.

\section{The effects of HPSE plasmid or shRNA on cell migration}

Cells were transiently transfected with HPSE plasmid or shRNA, and cell migration was observed by wound healing assay. The results showed that the wound healing rates were $17.7 \pm 1.5 \%, 16.0 \pm 1.6 \%$, $28.8 \pm 3.8 \%$ and $12.6 \pm 2.5 \%$ in control, empty vector, HPSE plasmid and shRNA transfection groups, respectively ( $p=0.00017$, Fig.4). These data confirmed that HPSE promoted cell migration, yet HPSE knockdown interfered with cell migration.

\section{The effects of HPSE plasmid or shRNA on cell invasion}

Cells were transiently transfected with HPSE plasmid or shRNA, and cell invasion was detected by transwell assay. $48 \mathrm{~h}$ after transfection, the number of invasive cells were $59.4 \pm 5.5$ and $54.8 \pm 4.3$ in cells of control and empty vector groups, respectively. However, the more invasive cells of $129.8 \pm 9.0$ in HPSE transfection group was observed, whereas downregulation of HPSE exerted the opposite effects with the less invasive cells of $38.6 \pm 5.6$ in HPSE knockdown group ( $p=0.00062)$, as shown in Fig.5. These results showed a cell invasion associated effect of HPSE.

\section{Effects of HPSE levels on the activations of signaling proteins}

Cells were transiently transfected with HPSE plasmid or shRNA, and multiple signaling molecules associated with cell proliferation (Akt/ERK), apoptosis (caspase-3/PARP), migration and invasion (FAK, PDK1/PKC/MARCKS) in transfected B-CPAP cells were detected by western blot. Our results showed that the phosphorylation of Akt/ERK was affected by HPSE upregulation and knockdown. The cleaved caspase-3/PARP were reduced in HPSE transfected cells, and found more active in HPSE knockdown cells. The activations of FAK and PDK1/PKC/MARCKS were promoted in cells with HPSE upregulation, yet repressed in cells with HPSE interference (Fig.6).

\section{Discussion}


HPSE is overexpressed in many types of human cancer and more expression of HPSE is associated with more tumor metastasis and invasion. Previous research has indicated that HPSE upregulation is correlated with poor prognosis and contributes to the lymphovascular invasion of breast cancer [9]. A systematic study showed that either the expression of HPSE in cancer and/or the serum concentration of HPSE may be a useful biomarker for the evaluation of surgery effects and prognosis prediction in ovarian cancer [10].

The mechanism(s) underlying tumor progression by HPSE have been shown variable. HPSE is associated with the aggressiveness of neuroblastoma, and Smad4 inhibits the tumorigenesis and progression of NB through suppressing the HPSE expression, through directly binding to its promoter and repressing the LEF1-facilitated transcription of HPSE. [11]. Downregulation of HPSE repressed glioma cell proliferation, whereas exogenous HPSE stimulated growth and activated ERK and AKT signaling. The anti-HPSE treatment of malignant glioma provides a novel strategy for the use of HPSE as a therapeutic target [12]. MiR-429 decreases the invasive capability by downregulating the expression of HSPE in gastric cancer cells [13]. Roneparstat, a non-anticoagulant heparin with potent anti-HPSE activity, inhibited the growth and brain metastatic breast cancer abilities of lapatinib-resistant cell clones. The inhibition of HPSEmediated signaling provides new insights to validate the use of Roneparstat for novel BMBC therapeutic strategies [14].

In this study, human papillary thyroid carcinoma of B-CPAP cells were transiently transfected with HPSE plasmid or shRNA to explore its effects on cell proliferation, apoptosis, migration and invasion. When HPSE was upregulated, cell proliferation was enhanced and apoptosis was inhibited. Cell migration and invasion were promoted. However, the downregulation of HPSE by specific shRNA transfection exerted the opposite effects. Our results are in agreement with previous findings in other human cancers, which indicated that HPSE facilitated the progression of papillary thyroid carcinoma.

To further investigate the probable cell signaling(s) downstream of HPSE, multiple antibodies of different signaling pathways were applied. Several studies have reported that ERK and Akt signaling were downstream of HPSE to facilitate cell proliferation. Differentiating Hpse-transgenic embryonic stem cells showed a higher growth rate, and overexpression of HPSE enhanced Erk and Akt phosphorylation in neural stem and progenitor cells [15]. The level of phosphorylated ERK and Akt was also found increased with HPSE transfection and decreased after HPSE knockdown in this study, which suggested that ERK and Akt were a part of HPSE signaling to mediate cell proliferation. It was reported that silencing HPSE expression activated apoptosis and autophagy of HCC cells [16]. Significantly increased apoptotic cells upon HPSE silencing was also found in melanoma cells, mediated by the activation of caspase 3/PARP1 pathway [17]. We showed here a negative correlation between the activities of cleaved caspase-3/PARP and the level of HPSE, which indicated that the caspase-3/PARP was probably involved in HPSE regulated cell apoptosis.

FAK were active signaling mediator in cell migration or invasion of various human cancers. FAK has previously been shown to be a downstream target of HPSE. Tumor cell invasiveness was reduced after 
knockdown of HPSE in myeloma and melanoma cells and lowering HPSE levels attenuates phosphorylation of FAK, a critical factor for the invasion of cancer cells. A study revealed the involvement of the HPSE-FAK axis in invasion of brain cancer cells [18]. 3-phosphoinositide-dependent protein kinase (PDK1) is participated in various cell signaling pathway, including Akt and PKC. Myristoylated alaninerich C-kinase substrate (MARCKS) is a substrate of PKC, activated in cell adhesion and motility. Molecular analysis suggested that PDK1 facilitated the progression of PIK3CA-driven head and neck squamous cell carcinoma (HNSCC). Both PIK3CA and PDK1 protein levels were correlated with tumor invasion and metastasis in human HNSCC clinical samples [19]. Knockdown of MARCKS suppressed cell migration and invasion of prostate cancer and facilitated cell-cell adhesion in cell colonies [20]. Our results showed that the activations of PDK1, PKC and MARCKS were induced after HPSE overexpression, and downregulated by HPSE knockdown. The phosphorylation of FAK was also altered by differential expression of HPSE. These findings revealed that multiple cell signaling pathways were affected by HPSE expression, and its complex effects on cell proliferation, apoptosis, migration and invasion caused by upor down-regulation would be further investigated.

\section{Conclusions}

Our data disclosed in cells of papillary thyroid carcinoma that multiple signaling molecules were involved in HPSE affected cell proliferation, apoptosis, migration and invasion. Therefore, our results revealed an initial insights for the activation of cell signaling induced by HPSE, which would provide possible therapeutic target to neutralize the effects of HPSE on papillary thyroid carcinoma. The specific inhibition for respective signaling pathway would be performed to further improve our conclusions, and the molecular mechanisms will be our main research direction.

\section{Abbreviations}

HPSE: heparanase; BCA: bicinchoninic acid; ECL: enhanced chemiluminescence; ERK: extracellular signalregulated kinase; FBS: fetal bovine serum; PDK1: 3-phosphoinositide-dependent protein kinase; PKC: protein kinase C; MARCKS: myristoylated alanine-rich C-kinase substrate; FAK: focal adhesion kinase; PARP: poly ADP-ribose polymerase

\section{Declarations}

\section{Acknowledgments}

Not applicable.

\section{Authors' contributions}


$\mathrm{CY}$ and $\mathrm{ZL}$ initiated and designed the study; $\mathrm{CY}, \mathrm{XC}$ and $\mathrm{YH}$ performed the experiments and wrote the manuscript; SZ contributed to the paper translation and gave experimental instructions; DC analyzed the data; ZL gave pertinent suggestions for the manuscript revision. All authors read and approved the final manuscript.

\section{Funding}

The study was supported by grants from the National Natural Science Foundation of China (No.81672644), given to the corresponding author Zhen Liu, who designed the study, interpreted the data and revised the manuscript.

\section{Availability of data and materials}

The datasets supporting the conclusions of the current study are available from the corresponding author on reasonable request. Please contact corresponding author, if you want to request the dataset.

\section{Ethics approval and consent to participate}

Not applicable.

\section{Consent for publication}

Not applicable.

\section{Competing interests}

The authors declare that they have no competing interests.

\section{Author details}

${ }^{1}$ Department of General Surgery, the Affiliated Shengjing Hospital of China Medical University, Shenyang 110004, China. 
${ }^{2}$ Department of Pathology, the Affiliated Shengjing Hospital of China Medical University, Shenyang 110004 , China.

${ }^{3}$ Science and Experiment Center, China Medical University, Shenyang 110122, China.

\section{References}

1. Zheng H, Ruan J, Zhao P, Chen S, Pan L, Liu J. Heparanase is involved in proliferation and invasion of ovarian cancer cells. Cancer Biomark. 2015;15(5):525-34.

2. Chen XP, Luo JS, Tian Y, Nie CL, Cui W, Zhang WD. Downregulation of Heparanase Expression Results in Suppression of Invasion, Migration, and Adhesion Abilities of Hepatocellular Carcinoma Cells. Biomed Res Int. 2015;2015:241983.

3. Lv B, Zhang B, Hu XY, Zeng QD. Heparanase regulates in vitro VEGF-C expression and its clinical significance to pancreatic ductal cell adenocarcinoma. Oncol Lett. 2016;11(2):1327-34.

4. Lv Q, Wu K, Liu F, Wu W, Chen Y, Zhang W. Interleukin-17A and heparanase promote angiogenesis and cell proliferation and invasion in cervical cancer. Int J Oncol. 2018;53(4):1809-17.

5. Chen X, Jiang W, Yue C, Zhang W, Tong C, Dai D, et al. Heparanase Contributes To Trans-Endothelial Migration of Hepatocellular Carcinoma Cells. J Cancer. 2017;8(16):3309-17.

6. Ilan N, Shteingauz A, Vlodavsky I. Function from within: Autophagy induction by HPSE/heparanasenew possibilities for intervention. Autophagy. 2015;11(12):2387-9.

7. Zheng $L$, Jiao $W$, Song $H$, Qu H, Li D, Mei H, et al. miRNA-558 promotes gastric cancer progression through attenuating Smad4-mediated repression of heparanase expression. Cell Death Dis. 2016;7(9):e2382.

8. Matos LL, Suarez ER, Theodoro TR, Trufelli DC, Melo CM, Garcia LF, et al. The Profile of Heparanase Expression Distinguishes Differentiated Thyroid Carcinoma from Benign Neoplasms. PLoS One. 2015;10(10):e0141139.

9. Tang D, Piao Y, Zhao S, Mu X, Li S, Ma W, et al. Expression and correlation of matrix metalloproteinase-9 and heparanase in patients with breast cancer. Med Oncol. 2014;31(7):26.

10. Zhang W, Chan H, Wei L, Pan Z, Zhang J, Li L. Overexpression of heparanase in ovarian cancer and its clinical significance. Oncol Rep. 2013;30(5):2279-87.

11. Qu H, Zheng L, Jiao W, Mei H, Li D, Song H, et al. Smad4 suppresses the tumorigenesis and aggressiveness of neuroblastoma through repressing the expression of heparanase. Sci Rep. 2016;6:32628.

12. Kundu S, Xiong A, Spyrou A, Wicher G, Marinescu VD, Edqvist PD, et al. Heparanase Promotes Glioma Progression and Is Inversely Correlated with Patient Survival. Mol Cancer Res. 2016;14(12):1243-53.

13. Sheng $N$, Zhang L, Yang S. MicroRNA-429 decreases the invasion ability of gastric cancer cell line BGC-823 by downregulating the expression of heparanase. Exp Ther Med. 2018;15(2):1927-33. 
14. Zhang L, Ngo JA, Wetzel MD, Marchetti D. Heparanase mediates a novel mechanism in lapatinibresistant brain metastatic breast cancer. Neoplasia. 2015;17(1):101-13.

15. Xiong A, Kundu S, Forsberg $M$, Xiong $Y$, Bergström $T$, Paavilainen T, et al. Heparanase confers a growth advantage to differentiating murine embryonic stem cells, and enhances oligodendrocyte formation. Matrix Biol. 2017;62:92-104.

16. Liu $\mathrm{M}$, Zhang $\mathrm{Y}$, Liao $\mathrm{Y}$, Chen $\mathrm{Y}, \mathrm{Pan} \mathrm{Y}$, Tian $\mathrm{H}$, et al. Evaluation of the Antitumor Efficacy of RNAiMediated Inhibition of CDC20 and Heparanase in an Orthotopic Liver Tumor Model. Cancer Biother Radiopharm. 2015;30(6):233-9.

17. Song T, Spillmann D. Transcriptomic analysis reveals cell apoptotic signature modified by heparanase in melanoma cells. J Cell Mol Med. 2019;23(7):4559-68.

18. Spyrou A, Kundu S, Haseeb L, Yu D, Olofsson T, Dredge K, et al. Inhibition of Heparanase in Pediatric Brain Tumor Cells Attenuates their Proliferation, Invasive Capacity, and In Vivo Tumor Growth. Mol Cancer Ther. 2017;16(8):1705-16.

19. Du L, Chen X, Cao Y, Lu L, Zhang F, Bornstein S, et al. Overexpression of PIK3CA in murine head and neck epithelium drives tumor invasion and metastasis through PDK1 and enhanced TGF $\beta$ signaling. Oncogene. 2016;35(35):4641-52.

20. Dorris E, O'Neill A, Hanrahan K, Treacy A, Watson RW. MARCKS promotes invasion and is associated with biochemical recurrence in prostate cancer. Oncotarget. 2017;8(42):72021-30.

\section{Figures}

A

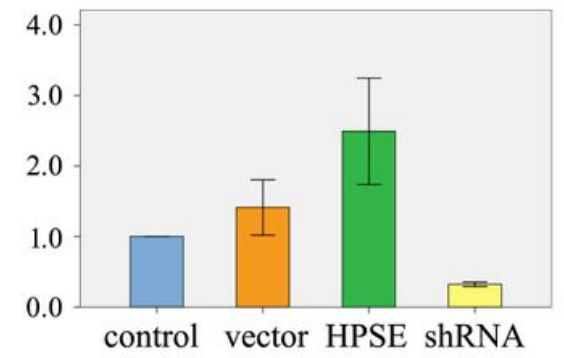

B

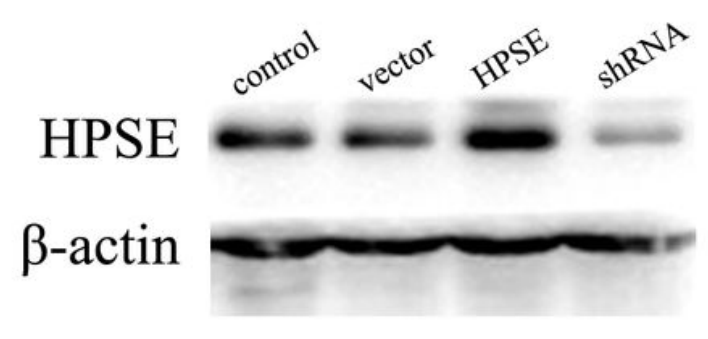

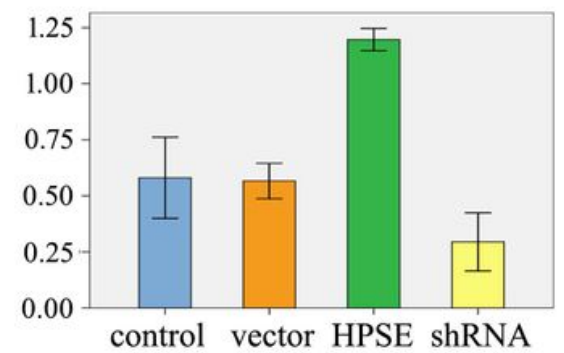

\section{Figure 1}

HPSE upregulation or knockdown was confirmed by qPCR (A) or western blot (B). A, The level of HPSE mRNA in HPSE transfected cells was upregulated, and that in shRNA transfected cells was less than control or empty vector group. B, The levels of HPSE protein in control, empty vector, HPSE or shRNA transfected cells. Statistical results showed that the level of HPSE protein in HPSE transfected cells was increased, and that in shRNA transfected cells was lower than control or empty vector group. 

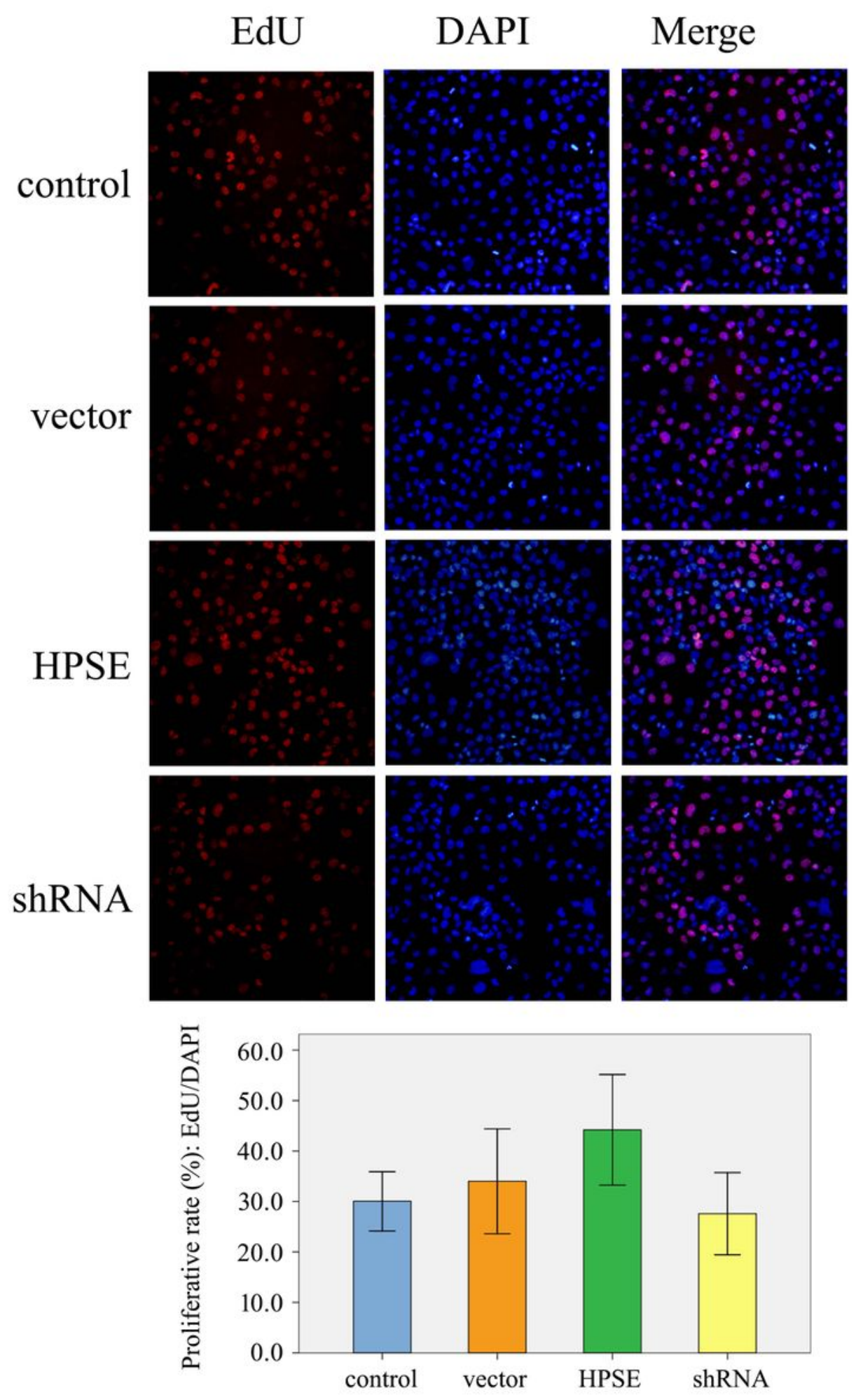

Figure 2

The cell proliferation of B-CPAP was inhibited by HPSE upregulation. The number of EdU positive cells was reduced in HPSE knockdown cells (Magnification 200x; EdU, red; DAPI, blue). 

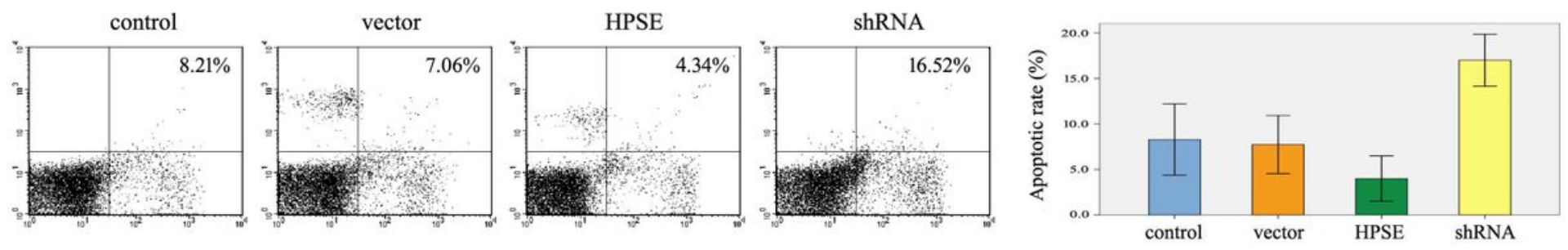

Figure 3

Cell apoptosis was detected by Annexin V-PE/7-AAD staining. Compared with control, empty vector and HPSE upregulation group, the apoptotic rate in HPSE knockdown cells was increased significantly. 

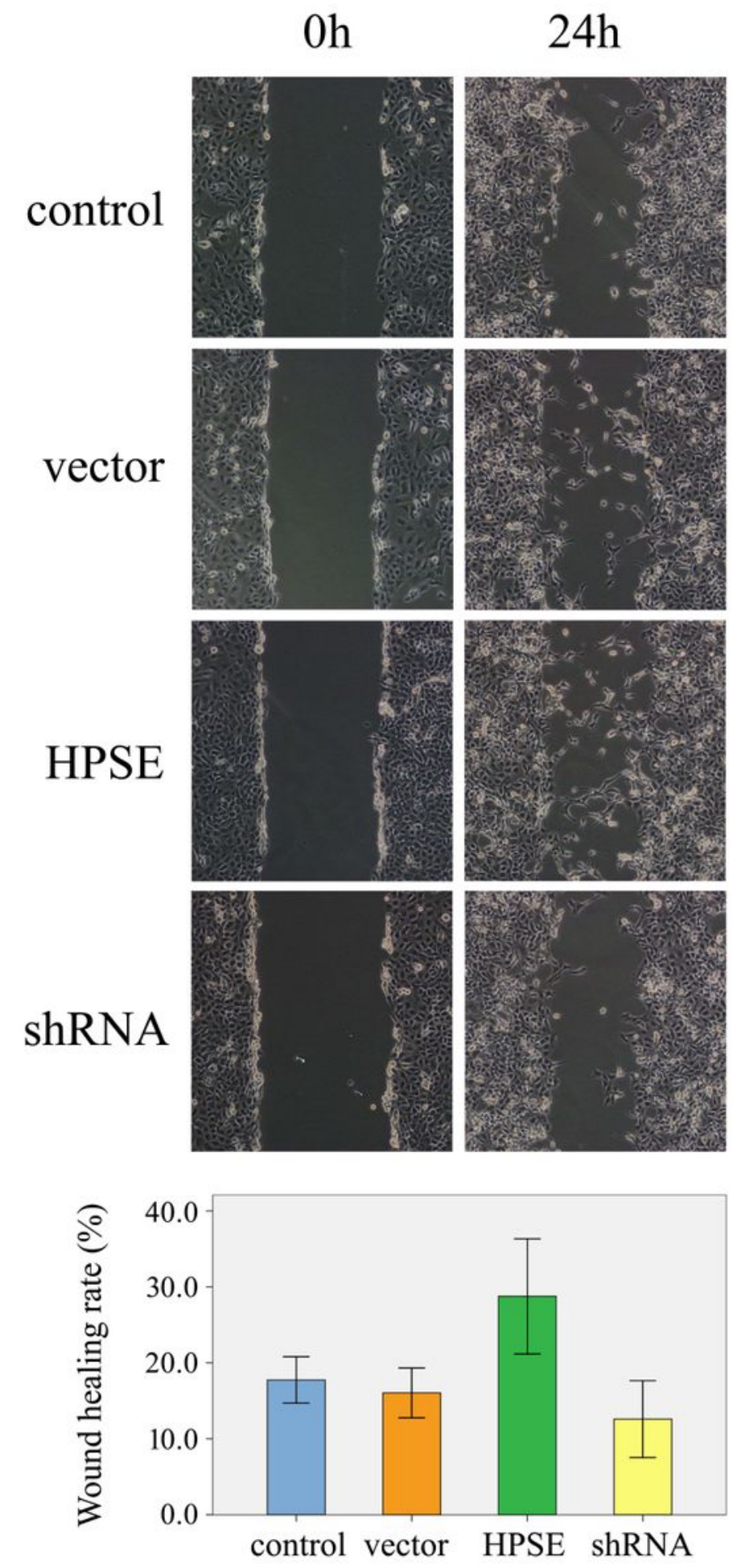

Figure 4

The wound healing rate was increased in HPSE transfected cells. The cell migration of B-CPAP was inhibited by HPSE knockdown (Magnification 100x). 


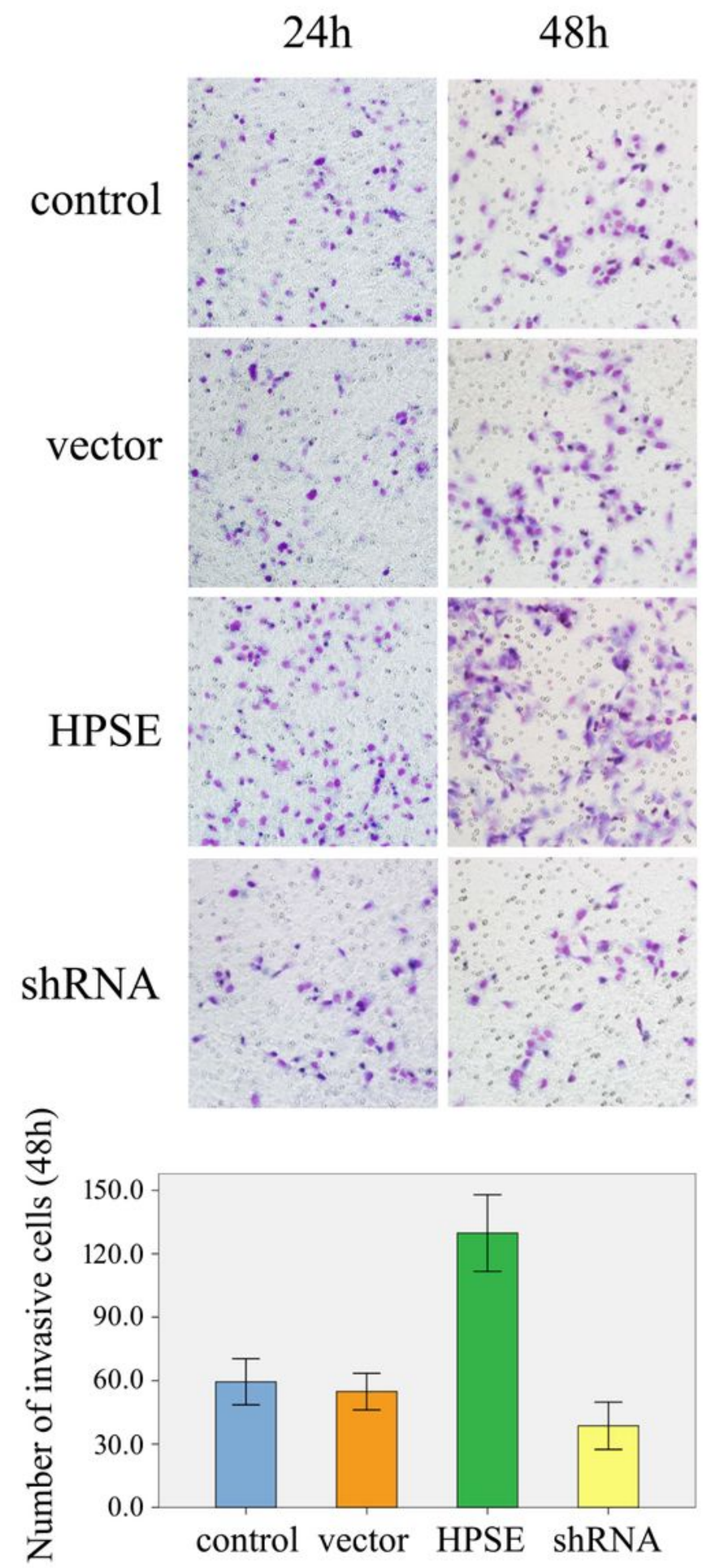

\section{Figure 5}

The number of invasive cells was increased in HPSE overexpression group. The cell invasion of B-CPAP was inhibited by HPSE shRNA (Magnification 200x; Giemsa staining). 


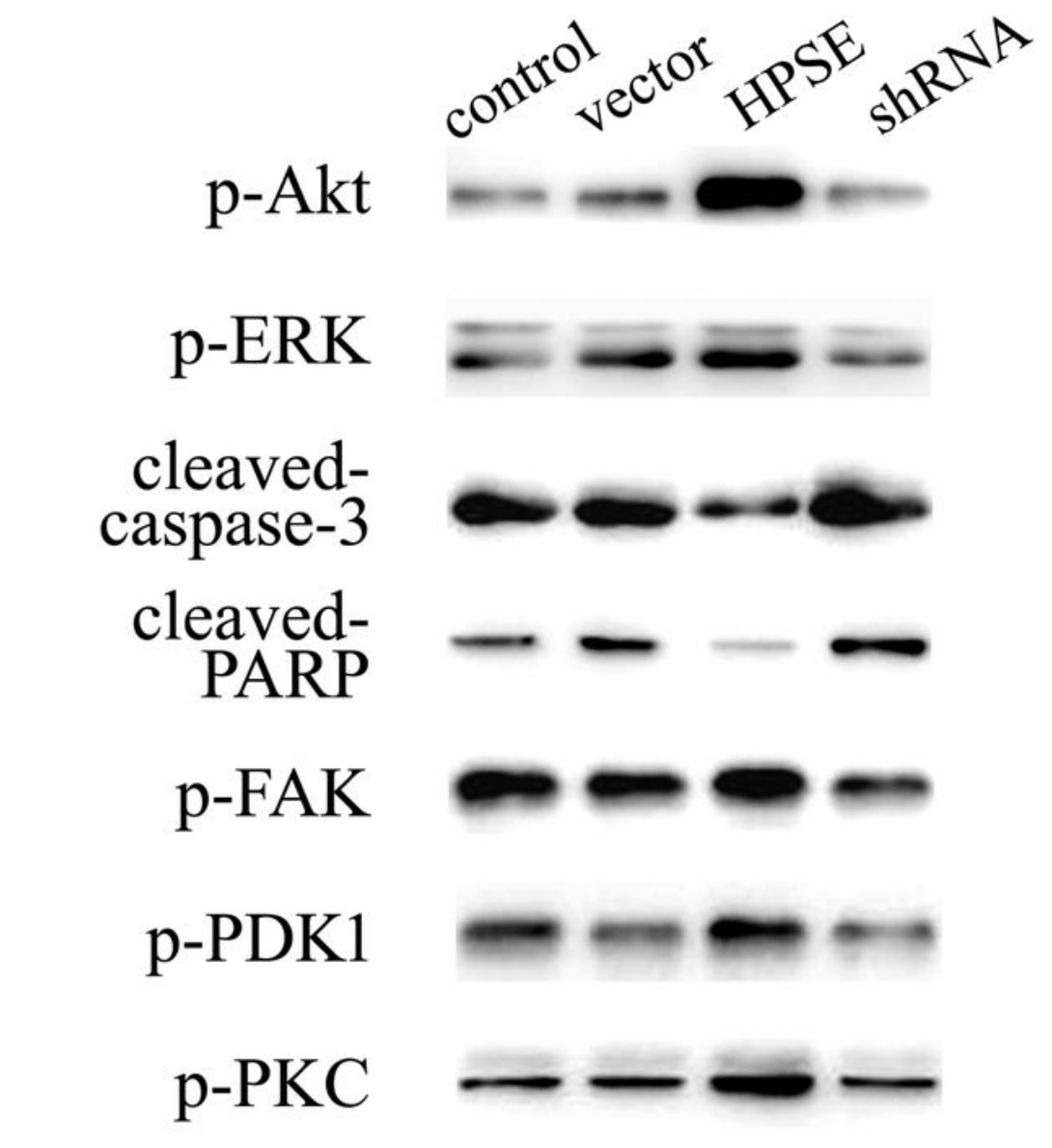

\section{p-MARCKS - - - -}

\section{$\beta$-actin}

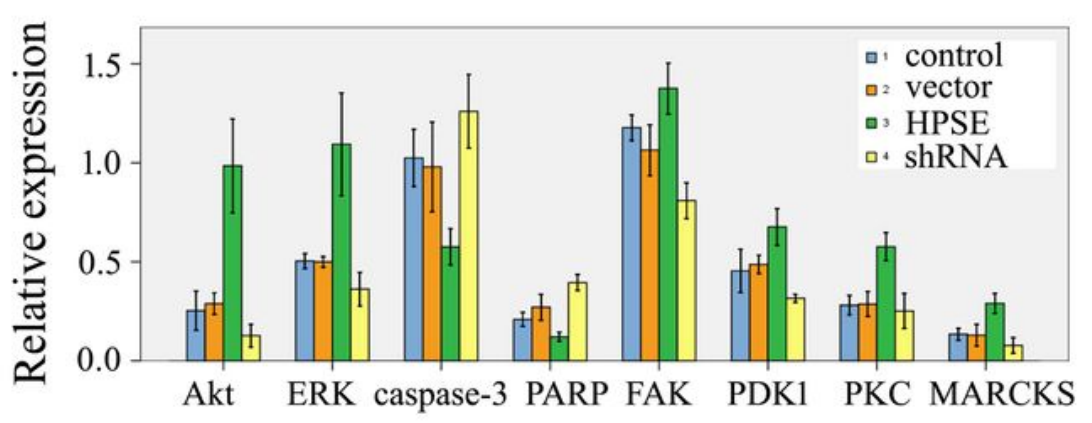

\section{Figure 6}

Effects of HPSE levels on the activations of signaling proteins associated with cell proliferation (Akt/ERK), apoptosis (caspase-3/PARP), migration and invasion (FAK, PDK1/PKC/MARCKS) in transfected B-CPAP cells. The phosphorylation of Akt/ERK was affected by HPSE upregulation and knockdown. The cleaved caspase-3/PARP were reduced in HPSE transfected cells, and found more active 
in HPSE knockdown cells. The activations of FAK and PDK1/PKC/MARCKS were promoted in cells with HPSE upregulation, yet repressed in cells with HPSE interference. 